\title{
CONSTRUCTION OF GLOBALLY CONVERGENT ITERATION FUNCTIONS FOR THE SOLUTION OF POLYNOMIAL EQUATIONS
}

\author{
BY J. F. TRAUB
}

Communicated by F. John, June 14, 1965

Iteration functions for the approximation of zeros of a polynomial $P$ are usually given as explicit functions of $P$ and its derivatives. We introduce a class of iteration functions which are themselves constructed according to a certain algorithm given below. The construction of the iteration functions requires only simple polynomial manipulation which may be performed on a computer.

Let $P$ be a real monic polynomial of degree $n$ with distinct zeros $\rho_{1}, \cdots, \rho_{n}$ and let the dominant zero $\rho_{1}$ be real. The theory may be extended to multiple zeros, dominant complex zeros, and subdominant zeros.

Let $B(t)$ be an arbitrary polynomial of degree at most $n-1$ with $B\left(\rho_{1}\right) \neq 0$. Define a sequence of polynomials of degree $n-1$ by

$$
\begin{array}{r}
G(0, t, B)=B(t), \quad G(\lambda+1, t, B)=t G(\lambda, t, B)-\alpha_{0}(\lambda) P(t), \\
\lambda=0,1, \cdots,
\end{array}
$$

where $\alpha_{0}(\lambda)$ is the leading coefficient of $G(\lambda, t, B)$. From the polynomial $G(\lambda, t, B) \equiv G_{1}(\lambda, t, B)$, form the polynomial $G_{p}(\lambda, t, B)$ for any positive integer $p$ by

$$
G_{p}(\lambda, t, B)=\sum_{k=0}^{p-1-k}[-P]^{p-1-k} \frac{G^{(p-1-k)}(\lambda, t, B)}{(p-1-k) !} V_{k}(t),
$$

where $V_{k}(t)$ is formed by

$$
V_{0}(t)=1, \quad V_{k}(t)=P^{\prime}(t) V_{k-1}(t)-\frac{P(t)}{k} V_{k-1}^{\prime}(t) .
$$

Define an iteration function for fixed $p$ and $\lambda$ by

$$
\phi_{p}(\lambda, t, B)=t-P(t) \frac{G_{p-1}(\lambda, t, B)}{G_{p}(\lambda, t, B)} .
$$

The global nature of the convergence is given by

TheOREM. Let $t_{0}$ be an arbitrary point in the extended complex plane such that $t_{0} \neq \rho_{2}, \rho_{3}, \cdots, \rho_{n}$ and let $t_{i+1}=\phi_{p}\left(\lambda, t_{i}, B\right)$. Then for all sufficiently large but fixed $\lambda$, the sequence $t_{i}$ is defined for all $i$ and $t_{i} \rightarrow \rho_{1}$. 
REMARK. Since $\phi_{p}\left(\lambda, \rho_{j}, B\right)=\rho_{j}, j=1,2, \cdots, n$, we have an:

Alternative Formulation. Let $t_{0}$ be an arbitrary point in the extended complex plane and let $t_{i+1}=\phi_{p}\left(\lambda, t_{i}, B\right)$. Then for all sufficiently large but fixed $\lambda$ the sequence $t_{i}$ is defined for all $i$ and $t_{i} \rightarrow p_{i}$ for some $j$.

Observe that the sequence is defined for all $i$. This should be compared with a sequence generated by, say the Newton-Raphson iteration function where the sequence is not defined at the zeros of $P^{\prime}$.

The asymptotic rate of convergence is given by

$$
\begin{aligned}
\lim _{t \rightarrow \rho_{1}} \frac{\phi_{p}(\lambda, t, B)-\rho_{1}}{\left(t-\rho_{1}\right)^{p}} & =C_{p}(\lambda, B), \\
C_{p}(\lambda, B) & =-\sum_{i=2}^{n} \frac{d_{i} \mu_{i}^{\lambda}}{\left(\rho_{1}-\rho_{i}\right)^{p-1}},
\end{aligned}
$$

where

$$
d_{i}=\frac{B\left(\rho_{i}\right)}{B\left(\rho_{1}\right)} \frac{P^{\prime}\left(\rho_{1}\right)}{P^{\prime}\left(\rho_{i}\right)}, \quad \mu_{i}=\frac{\rho_{i}}{\rho_{1}}, \quad i=2,3, \cdots, n .
$$

Hence the order of $\phi_{p}(\lambda, t, B)$ is $p$ while the asymptotic error constant (Traub $\left[1\right.$, p. 9]) is given by $C_{p}(\lambda, B)$. Observe that

$$
\lim _{\lambda \rightarrow \infty} C_{p}(\lambda, B)=0 \text {. }
$$

Results on the character of the convergence are readily available. An example is furnished by the following

Theorem. Let $\left|\rho_{1}\right|>\left|\rho_{2}\right|>\left|\rho_{j}\right|, j=3,4, \cdots, n$. Choose $p$ and $\lambda$ even and $B=P^{\prime}$. Let $\lambda$ be sufficiently large. Then if $\rho_{1}>\rho_{2}, t_{i} \uparrow \rho_{1}$; if $\rho_{1}<\rho_{2}, t_{i} \downarrow \rho_{1}$.

The iteration functions $\phi_{p}(0, t, 1)$ are classical. Calculating $\lim _{\lambda \rightarrow \infty} \phi_{p}(\lambda, \infty, B)$ or $\lim _{p \rightarrow \infty} \phi_{p}(0,0,1)$ are classical noniterative methods for approximating the zeros of polynomials.

The proofs of these and additional results will appear elsewhere.

\section{REFERENCE}

1. J. F. Traub, Iterative methods for the solution of equations, Prentice-Hall, Englewood Cliffs, N. J., 1964.

Bell Telephone Laboratories, Incorporated, Murray Hill, New Jersey 